FEBRUARY MEETING OF SAN FRANCISCO SECTION. 369

\title{
THE FEBRUARY MEETING OF THE SAN FRANCISCO SECTION.
}

THE eleventh regular meeting of the San Franciseo Section of the American Mathematical Suciety was held at Stanford University on February 23, 1907. The first session opened at eleven o'clock, with Professor E. J. Wilczynski, of the University of California, in the chair. The attendance was eighteen, including the following twelve members of the Society :

Professor R. E. Allardice, Professor H. F. Blichfeldt, Professor R. L. Green, Professor M. W. Haskell, Professor L. M. Hoskins, Professor D. N. Lehmer, Dr. W. A. Manning, Professor H. C. Moreno, Professor C. A. Noble, Dr. T. M. Putnam, Professor Irving Stringham, Professor E. J. Wilczynski.

The next meeting of the Section will be held at the University of California on September 28, 1907.

The following papers were read at the February meeting:

(1) Professor E. J. Wilczynski: "Projective differential geometry of curved surfaces."

(2) Professor H. F. Blichfeldt : "Concerning some properties of surfaces of revolution."

(3) Professor G. A. Miller : "On the groups generated by two operators of order three whose product is of order four."

(4) Professor L. M. Hoskins : "The practical formulation of the laws of motion."

(5) Professor R. E. Alladidice : "On a limit of the roots of an equation that is independent of all but two of the coefficients."

(6) Mr. E. T. BeLl : "Note on linear congruences."

(7) Dr. W. A. Manning: "On the limit of the degree of primitive groups."

Mr. Bell was introduced by Professor Blichfeldt. In the absence of Professor Miller, his paper was read by title. Abstracts of the papers follow below.

1. Professor Wilczynski's paper introduces a number of new conceptions into the theory of surfaces and traces their interrelations. One of the most interesting of these is as follows. Through every point $P$ of a surface $S$ there pass two asymptotic curves $A_{1}$ and $A_{2}$. Construct the locus of the asymptotic tan- 
gents of $S$ belonging to the points of $A_{1}$. We thus obtain, besides the developable $D_{1}$ of which $A_{1}$ is the cuspidal edge, a ruled surface $R_{1}$ upon which $A_{1}$ is an asymptotic line. Similarly for $A_{2}$. The osculating hyperboloids of $R_{1}$ and of $R_{2}$ at $P$ coincide. The osculating linear complexes of the four surfaces $P_{1}, R_{1}, D_{2}, R_{2}$ are so related that each is in involution with two of the others. Consider in particular the osculating linear complexes of $D_{1}$ and $D_{2}$. The directrices of the linear congruence common to them are of special interest. One of them lies in the plane tangent to $S$ at $P$, while the other passes through $P$. Since there are two such directrices associated with every point $P$ of $S$, there are thus obtained, associated with the surface $S$, two congruences. Consider for instance the congruence of the directrices of the first kind, those which pass through $P$. There are two directions through $P$ such that the directrices of the same kind belonging to points of $S$ infinitesimally close to $P$ in those directions will intersect the directrix of $P$. In this way two families of $\infty^{1}$ curves are defined on $S$, two passing through every point. These are called the directrix curves. The directrices of the second kind, treated from a similar point of view, yield the same curves on $S$.

The non-homogeneous equation of a non-ruled surface may be expressed by a development of the form

$$
z=x y+\frac{1}{6}\left(x^{3}+y^{3}\right)+\frac{1}{24}\left(I x^{4}+J y^{4}\right)+\cdots,
$$

where $I$ and $J$ are absolute projective invariants. The complete geometric interpretation of this canonical form is given and is found to depend upon the properties of certain cubic surfaces which have contact of the third order with $S$ at $P$, and which are completely determined by certain further geometric properties.

Analytically the treatment is based upon the theory of the invariants of a system of two partial linear differential equations of the second order with two independent variables.

2. Representing the plane conformally upon any surface, the straight lines of the plane will give rise to a system of curves on the surface, the study of which is interesting for the reason that they may be taken to represent straight lines in a plane geometry in which the euclidean axioms hold, with the exception of the "superposition" axiom. In particular, as Professor Blichfeldt points out, if the surface taken be a certain surface of revolution, some interesting results follow. 
3. Professor Miller's paper is devoted to the study of some of the groups which are generated by two operators $s_{1}, s_{2}$ of order three whose product is of order four. The main results are as follows: The order of the commutator of $s_{1}, s_{2}$ must always be greater than 2. If the order of $s_{1} s_{2}^{2}$ is 3 , the group $\left\{s_{1}, s_{2}\right\}$ generated by $s_{1}, s_{2}$ is the group of order 48 which involves operators of order 4 but no subgroup of order 24 . If two operators satisfy the conditions $s_{1}^{3}=s_{2}^{3}=\left(s_{1} s_{2}\right)^{4}=\left(s_{1} s_{2}^{2}\right)=1$, they generate the simple group of order 168 unless each of them is identity. If two operators whose commutator is of order 5 satisfy the conditions $s_{1}^{3}=s_{2}^{3}=\left(s_{1} s_{2}\right)^{4}=\left(s_{1} s_{2}^{2}\right)^{5}=1$, they generate the simple group of order 360 . When two operators of order 2 have a commutator of even order, they generate a group whose order is four times the order of their commutator; when they have a commutator of odd order, they generate a group whose order is either twice or four times the order of their commutator. If an operator of order 2 and an operator of order 3 have a commutator of order 2, they generate either the tetrahedral group or the direct product of this group and the group of order 2 . If two operators of order 3 have a commutator of order 2, they generate a group whose order is one of the four numbers 12, 36, 144, 288. The eight commutators which may be obtained from a single commutator by permuting its elements have a common order.

4. Professor Hoskins's paper is in abstract as follows: By a "practical" formulation of the laws of motion is meant a form of statement suited to the point of view of the average intelligent student pursuing a first course in mechanics. Such a formulation must not be palpably unsound, but neither can it aim at strict logical rigor. It must be based upon preliminary notions derived from ordinary experience, and must not raise highly abstract questions or logical subtleties which can be appreciated only by the exceptional mind and after mature study and reflection. The points especially emphasized in the paper are (1) that force should be taken as a fundamental quantity (contrary to the practice of some writers who aim at logical rigor); (2) that the simple fact that every force acting upon a body is exerted by some other body should not be left to inference but should be an explicit part of the definition of force, and should be further emphasized in the statement of Newton's first and third laws; (3) that the law of action and 
reaction should be restricted to forces, and should state explicitly that two bodies and only two are concerned in every "action and reaction"; and (4) that the law of composition of forces should be accepted as a fundamental principle, being neither implicitly contained in Newton's second law nor otherwise susceptible of deductive proof. This fourth point is urged both on the ground of intelligibility to the beginner and on that of scientific soundness. The paper does not attempt a full discussion of the question as to the best method of presenting an elementary course in mechanics. The formal statement of the laws of motion is not the best starting point for such a course, but on the contrary should come somewhat late. The practical value of such a formal statement is to sum up in convenient form what is already more or less familiar to the student.

5. Professor Allardice gave a proof of the following theorem: Every equation of the form $a x^{n}+b x^{m}+c x^{l}+\cdots+a_{1} x+a_{0}=0$ has a root whose modulus is not greater than

$$
\left|\frac{a_{0}}{a_{1}}\right| \cdot \frac{n}{n-1} \cdot \frac{m}{m-1} \cdot \frac{l}{l-1} \cdots
$$

whatever be the values of the coefficients $a, b, c, \cdots$.

6. Instead of proceeding as usual in the solution of a linear congruence in $n$ indeterminates, Mr. Bell makes use of the theory of partitions. The absolute term $c$ has added to it multiples of the modulus, and the resulting absolute terms $c_{0}, c_{1}, \cdots, c_{k}$ (where $c_{i}=c+i m+i$ ranges from 0 to the integral part of the fraction $(n m-c) / m$, where $m$ is the modulus) are partitioned in all possible ways into $m$ parts, and from the solution of $m$ congruences in one indeterminate the (incongruent) solutions of the original congruence are obtained. Further, the solutions fall into "classes," according to the value of $i$, and each "class" gives rise to a number of " sets" of solutions, the number of sets being the number of partitions of $c+i m$ into $n$ parts, no part being greater than $m$. The number of solutions of the original congruence in each "set" is easily determined and hence partition formulas for the number of solutions (incongruent, $\bmod m$ ) are obtained. These may be utilized to deduce theorems in partitions by equating them to the known formulas as given, e. g., by Smith ("Congruences and Equations," Col- 
lected Works, volume 1). Also the known criteria for the resolubility of such congruences can be put in partition forms.

7. The limit of the degree of a simply transitive primitive group which does not include the alternating group of that degree and which contains a given substitution is the object of investigation in Dr. Manning's paper. If the given substitution is of the (odd) prime order $p$ and of degree $p q$, such a limit is given by the formula

$$
(p+q)\left\{q+\frac{p-1}{p+1}+\mu(q-1)\right\}-\frac{p+\theta q}{\theta-1} \theta^{\mu},
$$

where $\theta=2 p /(p-1)$ and $\mu$ is the characteristic of $\log _{\theta} q$.

W. A. Manning,

Secretary of the Section.

\section{THE FIFTY-SEVENTH MEETING OF THE AMER- ICAN ASSOCIATION FOR THE ADVANCE- MENT OF SCIENCE.}

THe fifty-seventh meeting of the American Association for the Advancement of Science was held in New York City during the convocation week December 27, 1906, to January 2, 1907: The president of the meeting was Dr. W. H. Welch, of Johns Hopkins University. The address of the retiring president, Professor C. M. Woodward, entitled "The Science of Education," was given in the auditorium of the Horace Mann School of Columbia University on the evening of the opening day. This address has been published in full in Science for December 28, 1906.

The meetings of Section A were not well attended, only about twenty members being present at the organization meeting. The officers of the section were : vice-president, Edward Kasner ; secretary, L. G. Weld ; councilor, G. B. Halsted ; member of the general committee, James McMahon ; press secretary, the secretary of the section ; sectional committee, Ormond Stone, E. B. Frost, E. O. Lovett, Harris Hancock, A. $\mathrm{N}$. Skinner, together with the vice-president and the secretary of the section. 